\title{
Local vibrational density of states in disordered graphene
}

\author{
D.L. Kardashev ${ }^{1}$, K.D. Kardashev ${ }^{2}$ \\ ${ }^{1}$ National University "Odessa Maritime Academy” \\ 8, Didrikhson str., 65029 Odessa, Ukraine; e-mail: dlkardashev@ukr.net \\ ${ }^{2}$ A.S. Popov Odessa National Academy of Telecommunication \\ 1, Kuznechnaya str.,65029 Odessa,Ukraine; e-mail: konstantinkardashev@gmail.com
}

\begin{abstract}
Local vibrational density of states for disordered graphene has been calculated via Green's functions method. Disordered material has been modeled with Bethe lattice. Density of states does not include particularities specific for ideal graphene.
\end{abstract}

Keywords: Bethe lattice, Green function, local vibrational density of states.

Manuscript received 06.04.16; revised version received 14.07.16; accepted for publication 13.09.16; published online 04.10.16.

\section{Introduction}

The Green functions (GF) method has been successfully applied for investigation of electronic $[1,2]$ and dynamic $[3,4]$ properties of disordered solids $[5,6]$. It is impossible to use cyclic boundary conditions in these materials. In a real graphene, there are always some variations of chemical bounds lengths and valence angles magnitudes [5].

The most simple model of structurally disordered graphene is the Bethe lattice [7-9] (Cayley tree). It is an infinite connected cycle-free dendritic system of atoms with remaining short-range order. We've used lattice potential model in Born's approximation

$$
\begin{aligned}
& V(\vec{r})=\frac{1}{2} \beta \sum_{i, j}\left[\left(\vec{u}_{i}-\vec{u}_{i j}\right) \cdot \vec{r}_{j}(i)\right]^{2}+\frac{1}{4}(\alpha-\beta) \times \\
& \times \sum_{i j}\left(\vec{u}_{i}-\vec{u}_{i j}\right)^{2}+\frac{1}{4} \gamma \sum_{i j}\left[\left(\vec{u}_{i}-\vec{u}_{i j}\right) \times \vec{r}_{j}\right]^{2},
\end{aligned}
$$

The sums are on atoms $i$ and its nearest neighbors $j$, and $\mathbf{r}_{j}(i)$ is the unit vector joining they equilibrium positions; $\mathbf{u}_{i}$ and $\mathbf{u}_{j}-$ vectors of displacement of these atoms.

In order to determine GF, it is necessary to solve infinite sequence of Dyson's linear matrix equations:

$\left(m \omega^{2} \mathbf{I}_{i j}-\mathbf{D}_{0}\right) \mathbf{G}_{i j}=\mathbf{I}_{i j}+\sum_{v_{i} \ldots v_{j}} \mathbf{D}_{v_{1} \ldots v_{n}} \mathbf{G}_{i j}^{v_{i j} \ldots v_{j}}$.

Here, the sums are on all possible paths joining $i$-th and $j$-th atoms, $\mathbf{D}_{i j}$ - matrices of force constants.

The local density of states is given by

$$
g(E)=-\frac{2 \cdot \omega \cdot m}{3 \cdot \pi \cdot n} \sum_{k} \operatorname{Im} G_{k k}(\omega),
$$

where $n$ is a number of atoms in a system, and $G_{i i}(\omega)-$ diagonal matrix element of Green's function for $i$-th degree of freedom. Each summand is a partial density of vibrational states, which characterize contribution of a given degree of freedom with a frequency $\omega$ in the total density of states. 


\section{Solution for the Bethe lattice}

In order to transform graphene into the Bethe lattice, we use the following technique: any random atom we choose as initial and mark him with subscript 0 ; next nearest neighbors will have indexes 1, 2, etc. To investigate dynamics of this lattice, we postulate that each bond with a nearest neighbor can be characterized via $3 \times 3$ symmetric matrices of force constants $D_{i j}$ :

$$
\begin{aligned}
D_{01}=\left[\begin{array}{ccc}
\alpha & \beta & 0 \\
\beta & \alpha & 0 \\
0 & 0 & \gamma
\end{array}\right] ; \\
D_{02}=\left[\begin{array}{ccc}
\alpha-\frac{\sqrt{3} \cdot \beta}{2} & -\frac{\beta}{2} & 0 \\
-\frac{\beta}{2} & \alpha+\frac{\sqrt{3} \cdot \beta}{2} & 0 \\
0 & 0 & \gamma \\
D_{03} & {\left[\begin{array}{ccc}
\alpha+\frac{\sqrt{3} \cdot \beta}{2} & -\frac{\beta}{2} & 0 \\
-\frac{\beta}{2} & \alpha-\frac{\sqrt{3} \cdot \beta}{2} & 0 \\
0 & 0 & \gamma
\end{array}\right]}
\end{array}\right] .
\end{aligned}
$$

Then, the local GF of 0 -th atom can be determined from an infinite sequence of matrix equations [1,2]:

$$
\left\{\begin{array}{l}
\left(m \omega^{2} \mathbf{I}-\mathbf{D}_{\mathbf{0}}\right) \mathbf{G}_{\mathbf{0 0}}=\mathbf{I}+\sum_{j \neq 0} \mathbf{D}_{\mathbf{0 j}} \mathbf{G}_{\mathbf{0 j}} \\
\left(m \omega^{2} \mathbf{I}-\mathbf{D}_{\mathbf{0}}\right) \mathbf{G}_{\mathbf{0 i}}=\mathbf{D}_{\mathbf{0 i}} \mathbf{G}_{\mathbf{0 0}}+\sum_{j \neq 1} \mathbf{D}_{1 \mathbf{j}} \mathbf{G}_{1 \mathbf{j}} \\
\left(m \omega^{2} \mathbf{I}-\mathbf{D}_{\mathbf{0}}\right) \mathbf{G}_{2 \mathbf{i}}=\mathbf{D}_{1 \mathbf{i}} \mathbf{G}_{1 i}+\sum_{j \neq 2} \mathbf{D}_{2 \mathbf{j}} \mathbf{G}_{2 \mathbf{j}}
\end{array}\right.
$$

Here, $m$ is the mass of an atom, $\mathbf{I}$ - identity matrix, $\mathbf{D}_{\mathbf{0}}=\sum_{i=1}^{3} \mathbf{D}_{\mathbf{0} \mathbf{i}}=\left[\begin{array}{ccc}3 \alpha & 0 & 0 \\ 0 & 3 \alpha & 0 \\ 0 & 0 & 3 \gamma\end{array}\right]$ - force constants matrix joining atom with itself.

This system of equations can be solved, if one introduces the transfer matrices $\boldsymbol{\Phi}_{\mathrm{ji}}$ in the form:

$$
\left\{\begin{array}{l}
\mathbf{G}_{\mathbf{0 i}}=\boldsymbol{\Phi}_{0 \mathbf{i}} \mathbf{G}_{\mathbf{0 0}} \\
\mathbf{G}_{\mathbf{1 i}}=\mathbf{\Phi}_{1 \mathbf{i}} \mathbf{G}_{\mathbf{0 i}} \\
\cdot
\end{array}\right.
$$

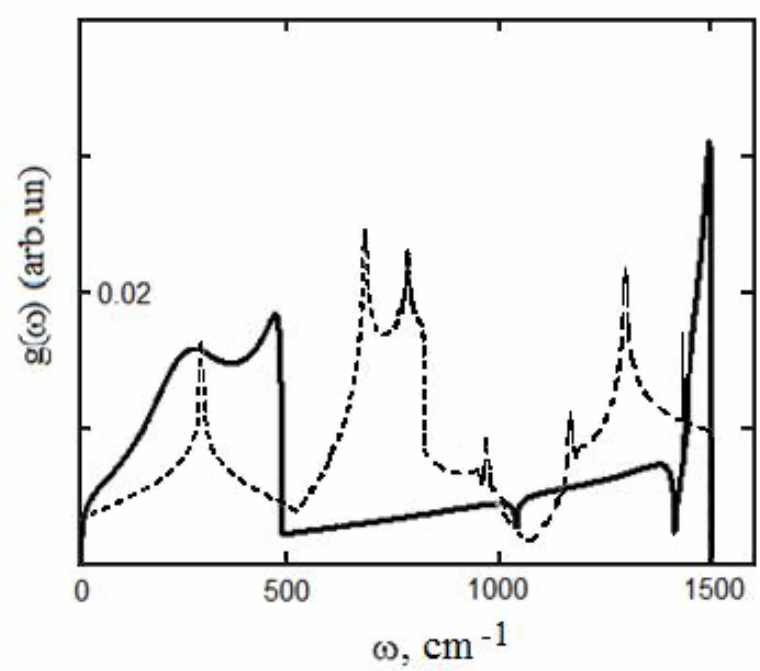

Figure. Local density of vibrational states of graphene. Solid line - density of states for the Bethe lattice, dashed line density of states of ideal graphene [10].

which satisfies equation

$$
\left(m \omega^{2} \mathbf{I}-\mathbf{D}_{\mathbf{0}}\right) \boldsymbol{\Phi}_{\mathbf{i j}}=\mathbf{D}_{\mathbf{i j}}+\sum_{k \neq j} \mathbf{D}_{\mathbf{i k}} \boldsymbol{\Phi}_{\mathbf{i k}} \boldsymbol{\Phi}_{\mathbf{k j}}
$$

Then, the Green function is determined as

$\mathbf{G}_{\mathbf{0 0}}=\left[m \omega^{2} \mathbf{I}-\mathbf{D}_{\mathbf{0}}-\sum_{i} \mathbf{D}_{\mathbf{0 i}} \mathbf{\Phi}_{\mathbf{0 i}}\right]^{-1}$.

Matrix elements were determined in an analytical form (here we does not perform them due to their massiveness).

The results of calculation of vibrational density of states for disordered graphene are represented in the figure. The dashed line represents density of states for ideal graphene [10]. Values of force constants have been selected due to the best proximity to X-ray inelastic scattering experimental data [11].

\section{Conclusions}

Local vibrational density of states describes well $\mathrm{ZO}$, ZA, LO, TO modes. But TA and LA peaks are highly smoothed.

In a presence of structural disorder, the expression for local density of states must be averaged over different local configurations.

The Bethe lattice can be used in a cluster modeling calculations, including presence of cycles of bonds, as boundary conditions.

\section{References}

1. S. Viola Kusminskiy, D.K. Campbell, and A.H. Castro Neto, Lenosky's energy and the phonon dispersion of graphene // Phys. Rev. B, 80, p. 035401-1-035401-5 (2009). 
2. M. Neek-Amal, Graphene nanoribbons subjected to axial stress // Phys. Rev. B, 82, p. 085432-1085432-6 (2010).

3. D. Cheliotis, B. Virág, The spectrum of the random environment and localization of noise // Probab. Theory Relat. Fields, 148, p. 141-158 (2010).

4. Z.H. Ni, T. Yu, Y.H. Lu, Y.Y. Wang, Y.P. Feng, Z.X. Shen, Uniaxial strain on graphene: Raman spectroscopy study and band-gap opening // ACS Nano, 2, p. 2301-2305 (2008).

5. S.Yu. Davydov, Model of adsorption on amorphous graphene // Semiconductors, 50(3), p. 377-383 (2016).

6. S.Yu. Davydov, On the density of states of disordered epitaxial graphene // Semiconductors, 49(5), p. 615-620 (2015).
7. Koh Wada, Takehiko Fujita, Takashi Asahi, Lattice Vibration of the Cayley Tree // Progr. Theor. Phys. 59, No. 4, p. 1101-1114 (1978).

8. H. Böttger, Principles of the Theory of Lattice Dynamics. Physik-Verlag, 1983.

9. R. Alben, D. Weaire, J.E. Smith, M.H. Brodsky, Vibrational properties of amorphous $\mathrm{Si}$ and Ge // Phys. Rev. B, 11, No.6, p. 2271-2295 (1975).

10. V. Adamyan, V. Zavalniuk, Phonons in graphene with defects // J. Phys: Condens. Matter, 24, 015402 (10 p.) (2011).

11. M. Mohr, J. Maultzsch, E. Dobardžić et al., Phonon dispersion of graphite by inelastic X-ray scattering // Phys. Rev. B, 76, 035439 (2007). 